\title{
MEASURES OF LINDELOF AND SEPARABILITY IN APPROACH SPACES
}

\author{
R. BAEKELAND \\ Department of Statistics \\ University of Ghent \\ Hoveniersberg 4 \\ 9000 Ghent \\ Belgium
}

\section{R. LOWEN}

Department of Mathematics

University of Antwerp

Groenenborgerlaan 171

2018 Antwerp

Belgium

(Received July 16, 1992 and in revised form August 10, 1993)

ABSTRAC' $T$. In this paper we introduce the notions of separability and Lindelöf in approach spaces and investigate their behaviour under products and subspaces.

KEY WORDS AND PHRASES. Topological categories, separability, Lindelöf, approach spaces.

\section{AMS SUBJECT CLASSIFICATION CODES 54D65, 54D30, 54E35.}

\section{INTRODUCTION.}

In [5], the measure of non-compactness which was introduced by $\mathrm{K}$. Kuratowski in [8], was put into the framework of so-called approach spaces, rephrased there as "measure of compactnesss". Approach spaces, as explained in detail in [6] provide us with a common supercategory of the categories TOP of topological spaces with continuous maps, and $\mathrm{p}-M E T^{\infty}$ of extended metric spaces and non-expansive maps. The advantage of doing this was not only that Kuratowski's rather peculiar measure of non-compactness was thus put into a canonical setting, and that this setting allowed for a unified treatment of compactness for topological spaces and of total boundedness for metric spaces, but also that it was thus possible to prove some fundamental relationships between the measure of compactness of a family of spaces and their productspace.

In further study of approach spaces, and their application, especially to the analytical study of spaces of probability measures [7], [1], it turns out to be indispensable also to have at our disposal a mechanism to measure the deviation an approach space may have from being Lindelö and from being separable. The purpose of this paper is to introduce such canonical measures and to study their basic properties. We pay particular attention to subspaces and products of metric spaces.

\section{PRELIMINARIES.}

We shall use the following symbols $\mathbf{R}_{+}:=\left[0, \infty\left[, \mathbf{R}_{+}^{*}:=\right] 0, \infty\left[\right.\right.$ and $\overline{\mathbf{R}}_{+}:=[0, \infty]$. If $A \subset X$ then $\Theta_{A}$ stands for the function $X \longrightarrow \overline{\mathbf{R}}_{+}$taking the value 0 in points of $\mathrm{A}$ and $\infty$ elsewhere. We put an $\bar{\uparrow}$ (respectively $\uparrow$ ) for an increasing (respectively a strictly increasing) function, system, sequence or whatever. We shall also use the symbols $\downarrow$ and $\bar{\downarrow}$ respectively for strict decreasing respectively decreasing functions, system, sequence or whatever.

We shall recall some definitions from [5] and [6]. 'An extended pseudo-quasi-metric (shortly extended p-q-metric space) is a pair (X,d) where $d: X \times X \longrightarrow \overline{\mathbf{R}}_{+}$fulfils:

(M1) $\{d=0\} \supset \triangle_{X}:=\{(x, x) \mid x \in X\}$

(M2) d fulfils the triangle inequality. 
The map $\mathrm{d}$ is then called an extended pseudo-quasi-metric (shortly extended-p-q-metric). Other properties $d$ may fulfil are:
(M3) $d$ is symmetric
(M4) $\{d=0\} \subset \triangle_{X}$
(M5) $d$ is finite.

If $\mathrm{d}$ fulfils also (M3) we drop "quasi-" ("q-"), if it fulfils (M4) we drop "pseudo-" ("p-") and if it fulfils (M5) we drop "extended". If $A \subset X$ then $d(A):=\sup \{d(a, b) \mid a, b \in A\}$ stands for the diameter of $\mathrm{A}$.

A map $\delta: X \times 2^{X} \longrightarrow \overline{\mathbf{R}}_{+}$is called a distance if it fulfils

(D1) $\forall A \in 2^{X}, \forall x \in X: x \in A \Rightarrow \delta(x, A)=0$

(D2) $\forall x \in X: \delta(x, \emptyset)=\infty$

(D3) $\forall A, B \in 2^{X}, \forall x \in X: \delta(x, A) \wedge \delta(x, B)=\delta(x, A \cup B)$

(D4) $\forall A \in 2^{X}, \forall x \in X, \forall \varepsilon \in \overline{\mathbf{R}}_{+}: \delta(x, A) \leq \delta\left(x, A^{(e)}\right)+\varepsilon$ where

$A^{(e)}:=\{x \mid \delta(x, A) \leq \varepsilon\}$.

A collection $(\Phi(x))_{x \in X}$ of ideals in $\overline{\mathbf{R}}_{+}^{X}$ is called an approach system if it fulfils

(A1) $\forall x \in X, \forall \phi \in \Phi(x): \phi(x)=0$

(A2) $\forall x \in X, \forall \phi \in \overline{\mathbf{R}}_{+}^{X}: \forall \varepsilon, N \in \mathbf{R}_{+}^{*}, \exists \phi_{\varepsilon}^{N} \in \Phi(x)$ :

$$
\phi_{e}^{N}+\varepsilon \geq \phi \wedge N \Rightarrow \phi \in \Phi(x)
$$

(A3) $\forall x \in X, \forall \phi \in \Phi(x), \forall N \in \mathbf{R}_{+}^{*}, \exists \phi^{\prime} \in \prod_{x \in X} \Phi(x), \forall z, y \in X$ :

$$
\phi^{\prime}(x)(z)+\phi^{\prime}(z)(y) \geq \phi(y) \wedge N .
$$

We shall denote an approach system by $(\Phi(x))_{x \in X}$ or shortly $\Phi$ if no confusion is possible. If $\Phi$ is an approach system then $\Lambda:=(\Lambda(x))_{x \in X}$ is called a basis or base for $\Phi$ if it fulfils

(B1) $\forall x \in X: \Lambda(x)$ is a basis for an ideal

(B2) $\forall x \in X: \Phi(x)=\hat{\Lambda}(x)$ where:

$$
\hat{\Lambda}(x):=\left\{\phi \mid \forall \varepsilon, N \in \mathbf{R}_{+}^{*}, \exists \psi \in \Lambda(x): \psi+\varepsilon \geq \phi \wedge N\right\} .
$$

Further [6] if $\Phi$ is an approach system on $\mathrm{X}$ then the map

$$
\delta_{\Phi}: X \times 2^{X} \longrightarrow \overline{\mathbf{R}}_{+}:(x, A) \longrightarrow \sup _{\phi \in \Phi(x) a \in A} \inf _{a \in A} \phi(a)
$$

is a distance on X. From $\delta$ a distance on $\mathrm{X}$ we can construct the approach system $\Phi_{\delta}$ defined by:

$$
\Phi_{\delta}(x):=\left\{\phi \mid \forall A \subset X: \inf _{a \in A} \phi(a) \leq \delta(a, A)\right\}
$$

for all $x \in X$. Further we have $\Phi_{\delta_{\sharp}}=\Phi$ and $\delta_{\Phi_{\boldsymbol{\sigma}}}=\delta$. A space with an approach system or a distance is called an approach space.

If $(\mathrm{X}, \Phi)$ and $\left(\mathrm{X}^{\prime}, \Phi^{\prime}\right)$ are approach spaces then a function $\mathrm{f}: X \longrightarrow X^{\prime}$ is called a contraction if it fulfils any of the following equivalent conditions [6]: 
(C1) $\forall x \in X, \forall \phi^{\prime} \in \Phi^{\prime}(f(x)): \phi^{\prime} \circ f \in \Phi(x)$

(C2) For any basis $\Lambda^{\prime}$ for $\Phi^{\prime}: \forall x \in X, \forall \psi^{\prime} \in \Lambda^{\prime}(f(x)): \psi^{\prime} \circ f \in \Phi(x)$

(C3) $\forall x \in X, \forall A \subset X: \delta^{\prime}(f(x), f(A)) \leq \delta(x, A)$.

Approach spaces and contractions constitute a topological category [6] denoted AP. TOP is reflectively and coreflectively embedded in AP by:

$$
(X, \mathbf{T}) \stackrel{\imath d_{x}}{\longrightarrow}\left(X, A_{t}(\mathbf{T})\right),
$$

where the approach system of $A_{t}(\mathrm{~T})$ is $\left(\Phi_{\mathbf{T}}(x)\right)_{x \in X}:=\{\nu \mid \nu(x)=0$, u.s.c. at $\mathrm{x}\}$ for all $x \in X$. The associated distance is given by $\delta_{\mathbf{T}}(x, A)=0$ iff $x \in \bar{A}$ and $\delta_{\mathbf{T}}(x, A)=\infty$ iff $x \notin \bar{A}$ for all $x \in X, A \subset X$. Given $(X, \Phi) \in|A P|$ its TOP-coreflection is given by:

$$
\left(X, \mathbf{T}^{*}(\Phi)\right) \stackrel{\imath d x}{\longrightarrow}(X, \Phi),
$$

where $T^{*}(\Phi)$ is the topology determined by the neighborhood system:

$$
\left.N^{*}(\Phi)(x):=\left\{\{\nu<\varepsilon\} \mid \nu \in T^{\prime} r\right), \varepsilon \in \mathbf{R}_{+}^{*}, x \in X\right\}
$$

$\mathrm{T}^{*}$ is left inverse, right adjoint to $A_{\mathrm{t}}$.

We say that the approach space has property $\mathrm{P}$ iff the topological bicoreflection of this space has the topological property $\mathrm{P}$ (e.g. compact, Lindelö, ...). Analogously $p-q-M E T^{\infty}$ is bicoreflectively embedded in AP by:

$$
\begin{aligned}
p-q-M E T^{\infty} & \stackrel{A_{m}}{\longrightarrow} A P \\
(X, d) & \longrightarrow\left(X, A_{m}(d)\right),
\end{aligned}
$$

where $A_{m}(d)$ is determinded by the approach system $\left(\Phi_{d}(x)\right)_{x \in X}$ with $\Phi_{d}(x):=\{\nu \mid \nu \leq d(x,)$. for all $x \in X$. In this case the associated distance is given by $\delta_{d}(x, A)=\inf _{a \in A} d(x, a)$ for all $x \in X, A \subset X$. Given the approach space $\mathrm{X}$ with approach system $\Phi$ its $p-q-M E T^{\infty}$-coreflection is given by:

$$
(X, M(\Phi)) \stackrel{\text { ddx }}{\longrightarrow}(X, \Phi)
$$

where $M(\Phi)$ is the $\infty-p-q-$ metric defined by $M(\Phi)(x, y):=\delta_{\Phi}(x,\{y\})$. M is of course left inverse, right adjoint to $A_{m}$. Approach spaces for which $\delta\left(X \times 2^{X}\right)=\{0, \infty\}$ are topological [6].

If $\Lambda$ is a basis for the approach space $(X, \Phi)$ then:

$$
\delta_{\Phi}(x, A):=\sup _{\Psi \in \Lambda(x)} \inf _{a \in A} \Psi(a) .
$$

3 MEASURES OF SEPARABILITY AND LINDELOF.

We now introduce the measures of separability and Lindelö:

DEFINITION 3.1 If $(X, \Phi)$ is an approach space then we define the measure of Lindelof (respectively separability) of $(X, \Phi)$ as

$$
L(X):=\sup _{\phi \in \prod_{\boldsymbol{x} \in X}} \inf _{\Phi(x)} \sup _{Y \in 2^{\prime(x))}} \inf \phi(x)(z) .
$$

(respectively as

$$
\left.S(X):=\inf _{\boldsymbol{A} \in \mathbf{2}((x)))} \sup _{\mathbf{z} \in X} \delta(z, A)\right) .
$$

The following result is a straightforward exercise in topology:

LEMMA 3.2 A topological space $X$ is Lindelöf iff for every family $\left(V_{x}\right)_{x \in X}$ where $V_{x}$ is a neighborhood of $x$ there exists a countable set $\left\{x_{n} \mid n \in \mathbf{N}\right\}$ such that: $\bigcup_{n \in \mathbf{N}} V\left(x_{n}\right)=X$. 
Further

PROPOSITION 3.3 An approach space $X$ is separable iff $S(X)=0$.

Proof. It is clear that $\mathrm{S}(\mathrm{X})=0$ iff we can find an $A \in 2^{((X))}$ such that: $\sup _{x \in X} \delta(x, A)=0$ iff $\forall x \in X: \delta(x, A)=0$ iff $\forall x \in X: x \in \bar{A}$. So $\mathrm{S}(\mathrm{X})=0$ iff $\left(\mathrm{X}, \mathcal{T}^{\bullet}(\Phi)\right)$ is separable. ।

This result is not true for the Lindelof measure, as the following example shows.

COUNTEREXAMPLE 3.4 Consider $d_{<}: \mathbf{R} \times \mathbf{R} \rightarrow \overline{\mathbf{R}}$ whereby $d_{<}(a, b):=b-a$ if $a \leq b$ and $d_{<}(a, b):=\infty$ if $a>b$. It is easy to see that this defines a $p-q-M E T^{\infty}$ space on $\mathbf{R}$ Its topological bicoreflection is the RHO topology [3]. We now consider the product of this approach space with itself. It is not hard to see that $L(\mathbf{R} \times \mathbf{R})=0$. However since a coreflection preserves products, it follows from [3] that the space is not Lindelöf.

THEOREM 3.5 For a topological approach space $X$ we have:

$$
L(X), S(X) \in\{0, \infty\}
$$

Further:

$$
\begin{aligned}
& \text { (a) }(X, \mathcal{T}) \text { is separable iff } S(X)=0 \\
& \text { (b) }(X, \mathcal{T}) \text { is Lindelöf iff } L(X)=0
\end{aligned}
$$

Proof. It is clear that we also have:

$$
L(X):=\sup _{\psi \in \prod \prod_{x \in X} \wedge(x)} \inf _{\left.Y \in 2^{(}(x)\right)} \sup _{x \in X} \inf \psi(x)(z)
$$

where $\Lambda(x)$ is a basis of $\Phi(x)$. If we take:

$$
\Lambda(x)=\left\{\Theta_{V} \mid V \text { is an neighborhood of } x \text { in } \mathcal{T}\right\}
$$

then it is clear that $\mathrm{L}(\mathrm{X})$ can only have the values 0 or $\infty$. Because $\delta(x, A)$ can only have the values 0 or $\infty$ it is clear that $S(X)$ can only take these values too. To prove $(a)$ we only have to apply 3.3 , and (b) follows easily from 3.2 and expression ( 5).

In metric spaces we know that separability and Lindelöf coincide. We shall prove that the measures of separability and Lindelöf also coincide in p-MET

LEMMA 3.6 For the $p-q-M E T^{\infty}$ space $(X, d)$ we have:

$$
\begin{aligned}
& L(X)=\sup _{x \in X} \inf d(y, x) \\
& S(X)=\sup _{x \in X} \inf _{\boldsymbol{x} \in A} d(x, y)
\end{aligned}
$$

for a certain $A \in 2^{((X))}$.

Proof. It is clear from the definition that for any $A \in 2^{((x))}$ :

$$
L(X) \geq \sup _{x \in X} \inf d(y, x) .
$$

Further it is clear that for each $n \in N$ we can choose a set $A_{n} \in 2^{((X))}$ such that:

$$
\sup _{x \in X} \inf _{y \in A_{n}} d(y, x)>L(X)-\frac{1}{n} .
$$

And thus:

$$
L(X)=\sup _{x \in X} \inf _{\nu \in A} d(y, x)
$$

where $A=\bigcup_{n \in N} A_{n}$. The equality for $\mathrm{S}(\mathrm{X})$ is shown in a similar way. 
COROLlARY 3.7 For a $p$-MET space $(X, d)$ we have: $L(X)=S(X) \quad$ I.

In counterexample 5.1 we sec that for a general p-q- $M E T^{\infty}$ space the previous result is not true.

COROLLARY 3.8 An extended pseudometric space $X$ is separable iff $S(X)=L(X)=0$.

It is easy to see that for contractions we have the following:

THEOREM 3.9 For $X, X^{\prime} \in|A P|$ and $f: X \longrightarrow X^{\prime}$ a contraction :

$$
\begin{aligned}
& \text { (a) } L(f(X)) \leq L(X) \\
& \text { (b) } S(f(X)) \leq S(X) \text {. । }
\end{aligned}
$$

In topological spaces we can state the Lindelö property by means of filters with the countable intersection property [3]. We can do the same here for approach spaces. We shall put $F(X)$ (respectively $F_{\omega}(X)$ ) for the set of filters (respectively the set of filters with the countable intersection property) and analogously as for a filter we shall say that a base $\mathcal{B}$ of a filter $\mathcal{F} \in F(X)$ has the countable intersection property if for all $\left(B_{n}\right)_{n \in \mathbf{N}} \in \mathcal{B}$ :

$$
\bigcap_{n} B_{n} \in \mathcal{B}
$$

Obviously if a filter $\mathcal{F}$ has a base with the countable intersection property then the filter $\mathcal{F}$ itself has the countable intersection property.

We are now ready to prove the following theorem:

THEOREM 3.10 For $(X, \Phi) \in|A P|$ with base $(\Lambda(x))_{x \in X}$ we have:

Proof. First consider $Y \in 2^{((X))}$ and put:

$$
L(X)=\sup _{\mathcal{F} \in F_{\omega}(X)} \inf _{x \in X} \alpha \mathcal{F}(x) .
$$

$$
B_{Y}=\left\{x \in X \mid \exists \phi \in \prod_{z \in X} \Phi(z), \forall y \in Y: \phi(y)(x)>L(X)-\varepsilon\right\}
$$

The definition of $\mathrm{L}(\mathrm{X})$ implies that $B_{Y} \neq \emptyset$ and since $\bigcap_{n \in \mathrm{N}} B_{Y_{n}}=B_{\cup_{n} Y_{n}}, \mathcal{B}_{\varepsilon}=\left\{B_{Y} \mid Y \in 2^{((X))}\right\}$ is a base with the countable intersection property. Now consider the filter $\mathcal{F}_{e}:=<\mathcal{B}_{e}>$. Then:

$$
\begin{aligned}
& \inf _{z \in X} \alpha \mathcal{F}_{e}(z)=\inf _{z \in X} \sup _{\phi \in \Phi(z)} \sup _{B \in B_{c}} \inf \phi(x) \\
& =\sup _{\phi \in \prod_{x \in X}} \inf _{\Phi(x)} \sup _{\boldsymbol{x} \in X} \inf _{B \in B_{s}} \phi(z)(x)
\end{aligned}
$$

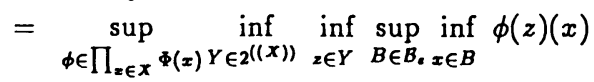

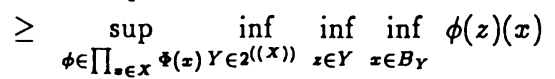

$$
\begin{aligned}
& \geq L(X)-\varepsilon \text {. }
\end{aligned}
$$

From the arbitrariness of $\varepsilon$ we deduce that:

$$
\sup _{\mathcal{F} \in F_{\omega}(X)_{z} \in X} \inf _{\alpha} \alpha \mathcal{F}(z) \geq L(X) .
$$

Second to prove the other inequality we first prove the following assertion:

Assertion: For every $F \in F_{\omega}(X)$ and $Y \in 2^{((X))}$ :

$$
\inf _{\boldsymbol{y} \in Y} \sup _{F \in \mathcal{F}} \inf _{\boldsymbol{z} \in F} \phi(y)(z) \leq \sup _{x \in X} \inf _{\boldsymbol{y} \in Y} \phi(y)(x) .
$$


Indeed put

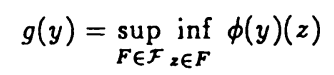

then $\exists F_{\nu} \in \mathcal{F}$ such that $\forall z \in F_{\nu}: \phi(y)(z)>g(y)-\varepsilon$. Because $\mathcal{F} \in F_{\omega}(X)$ we can find $z \in \bigcap_{\nu \in Y} F_{\nu}$. Hence,

$$
\phi(y)(z) \geq g(y)-\varepsilon,
$$

and thus;

$$
\inf _{\nu \in Y} \phi(y)(z)>\inf _{y \in Y} g(y)-\varepsilon
$$

So finally we obtain:

$$
\sup _{\boldsymbol{x} \in X} \inf _{\boldsymbol{y} \in Y} \phi(y)(x) \geq \inf _{\boldsymbol{y} \in Y} \sup _{\boldsymbol{F} \in \mathcal{F}_{\boldsymbol{z}} \in \boldsymbol{F}} \inf _{\boldsymbol{x}} \phi(y)(z)-\varepsilon .
$$

Since this is true for every $\varepsilon>0$ this proves the assertion.

Now take $\mathcal{F} \in F_{\omega}(X)$, then

$$
\begin{aligned}
& \inf _{x \in X} \alpha \mathcal{F}(x)=\inf _{x \in X} \sup _{\phi \in \Phi(x)} \sup _{F \in \mathcal{F}} \inf _{\boldsymbol{y} \in F} \dot{\phi}^{\prime}(y) \\
& =\sup _{\phi \in \prod_{x \in X}} \inf _{\boldsymbol{\phi}(\boldsymbol{x})} \sup _{\boldsymbol{x} \in \boldsymbol{X}} \inf \phi(x)(y)
\end{aligned}
$$

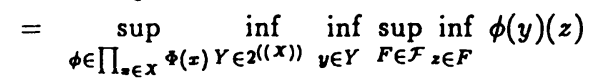

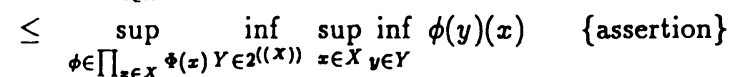

$$
\begin{aligned}
& =L(X) \text {. } \mathbf{I}
\end{aligned}
$$

\section{PRODUCTS.}

In this section we shall discuss the relations between the measures of Lindelof and separability of a product space and its component spaces.

REMARK 4.1 Since the projections are contractions it is clear from Theorem 3.9 that the measures of separability and Lindelof of the components are always less than or equal to the corresponding measure for the product space. So we only have to prove one equality for each of the measures.

\section{MeASURE OF LINDELÖF.}

Since the real line with the right half-open topology is Lindelöf and the product with itself is not Lindelö [3], Theorem 3.5 allows us to conclude that the product of approach spaces can have measure of Lindelöf equal to $\infty$ while the measures of Lindelöf for the components are 0 .

\section{Measure of Separability.}

For topological spaces [2] the product of separable spaces is separable iff the cardinality of the index set is less than or equal to the continuum. Along the same lines we obtain:

THEOREM 4.2 For the approach spaces $X_{i} \in|A P|, i \in I$ where $|I| \leq\left|2^{\mathrm{N}}\right|$ we have:

$$
S\left(\prod_{i \in I} X_{\mathbf{i}}\right)=\sup _{i \in I} S\left(X_{\mathbf{i}}\right) \text {. }
$$

Proof. Consider the set $A_{i}:=\left\{a_{i}(n) \mid n \in \mathrm{N}\right\} \subset X_{i}$ :

$$
\sup _{x_{i} \in X_{i} \phi_{i} \in \Phi_{1}\left(x_{i}\right)} \inf _{n \in N} \phi_{i}\left(a_{i}(n)\right)<S\left(X_{i}\right)+\varepsilon
$$


Because $|I| \leq\left|2^{\mathrm{N}}\right|$ there exists an injection $\varphi: I \rightarrow[0,1]$. We shall note $K=\varphi(I)$ and $\operatorname{CIR}_{[0,1]}$ for the set of closed intervals with rational endpoints in $[0,1]$. For each $\left\{J_{1}, J_{2}, \ldots, J_{k}\right\} \subset \mathcal{C} \mathcal{I R}_{[0,1]}$ and $n_{1}, \ldots, n_{k}$ we define the point $t\left(J_{1}, \ldots, J_{k}, n_{1}, \ldots, n_{k}\right)$ such that $t\left(J_{1}, \ldots, J_{k}, n_{1}, \ldots, n_{k}\right)_{l}=a_{l}\left(s_{l}\right)$ where $s_{l}=n$, if $\varphi(l) \in J, \cap K$ and $s_{l}=0$ if $\varphi(l) \notin \bigcup_{j=1, \ldots, k} J_{j} \cap K$. Because the set $\mathcal{C I R}_{\{0,1\}}$ is clearly countable the sct

$$
\Lambda:=\left\{t\left(J_{1}, \ldots, J_{k}, n_{1}, \ldots, n_{k}\right) \mid\left\{J_{1}, J_{2}, \ldots, J_{k}\right\} \subset \mathcal{C I T}_{[0,1]} \text { and } n_{1}, n_{2}, \ldots, n_{k} \in \mathbf{N}\right\}
$$

is also countable. Now consider $x \in \prod_{1 \in I} X_{1}$ and $\phi \in \Lambda(x)=\left\{\sup _{1 \in L} \zeta_{1}\left(p r_{1}().\right) \mid L \in 2^{(I)}\right.$ and $\left.\zeta_{1} \in \phi_{1}\left(x_{1}\right)\right\}$. Then with $L:=\left\{l_{1}, l_{2}, \ldots, l_{k}\right\}$ for each $\jmath=1, \ldots, k$ there exists a set $J_{j} \in \mathcal{C I R}_{[0,1]}$ : $\varphi\left(l_{3}\right) \in J$, where all $J$, can be taken pairwise disjoint. It is clear from Equation 9 that we can find for each $l$, a $n$, such that:

$$
\zeta_{l_{j}}\left(a_{l},\left(n_{j}\right)\right)<S\left(X_{l_{j}}\right)+\varepsilon
$$

It is now clear that the point $t:=t\left(J_{1}, \ldots, J_{k} ; n_{1}^{\prime}, \ldots, n_{k}\right)$ fulfills:

$$
\sup _{i \in L} \zeta_{\mathbf{i}}\left(p r_{\mathbf{\imath}}(t)\right)<\sup _{i \in I} S\left(X_{\mathbf{\imath}}\right)+\varepsilon . \quad \text { । }
$$

This theorem cannot be improved. Indeed take $|\chi|>\left|2^{\mathrm{N}}\right|$ and consider the set $\mathrm{N}^{x}$ with the product topology. The set $\mathrm{N}$ is clearly a separable topological space but it is well known ([9] example 103) that $\mathrm{N}^{x}$ is not separable. From Theorem 3.5 we now conclude that $S(N)=0$ but $S\left(N^{x}\right)=\infty$.

\section{SUBSPACES OF P-Q-MET ${ }^{\infty}$ SPACES.}

In this section we discuss some properties of the measures of separability and Lindelof in p-q- $M E T^{\infty}$ spaces, especially for subsets and products.

THEOREM 5.1 For a $p-M E T^{\infty}$ space $X$ and $Y \subset X$ we have:

$$
\begin{aligned}
& \text { (a) } L(Y) \leq 2 L(X) \\
& \text { (b) } S(Y) \leq 2 S(X) .
\end{aligned}
$$

Proof. (a) We know that:

$$
L(X)=\sup _{x \in X} \inf d(y, x) .
$$

for a certain $A \in 2^{((X))}$ and we write $A \cap Y=\left\{b_{n} \mid n<i n N\right\}$ and $A \cap Y^{c}=\left\{c_{n} \mid n \in \mathrm{N}\right\}$ if one of the sets is empty or finite we adjust the indexset ]. Suppose that there is a $y \in Y$ and an $m \in \mathbf{N}: \inf _{k \in \mathbf{N}} d\left(b_{k}, y\right)>L(X)+1 / m$, then there exists a $c_{n}: d\left(c_{n}, y\right)<L(X)+1 / m$. Consider now the sets:

$$
B_{n, m}=\left\{y \in Y \mid \inf _{k \in \mathbb{N}} d\left(b_{k}, y\right)>L(X)+1 / m \text { and } d\left(c_{n}, y\right)<L(X)+1 / m\right\} .
$$

From each non-empty set $B_{n, m}$ we choose exactly one $y_{n, m}$ (if this set is empty choose an element at random) and consider the set:

$$
B=\left\{y_{n, m} \mid n, m \in \mathrm{N}\right\} \cup\left\{b_{n} \mid n \in \mathrm{N}\right\}
$$


For $y \in Y$ two situations can occur. Either $\inf _{n \in N} d\left(b_{n}, y\right) \leq L(X)$, which gives us $\inf _{b \in B} d(b, y) \leq$ $L(X)$ or this is not true and then $\inf _{n \in \mathbf{N}} d\left(b_{n}, y\right)>L(X)+1 / m$, for some $m>0$ and so $\exists n \in \mathbf{N}$ : $d\left(c_{n}, y\right)<L(X)+1 / m$ which implies:

$$
d\left(y_{n, m}, y\right) \leq d\left(y_{n, m}, c_{n}\right)+d\left(c_{n}, y\right) \leq 2 L(X)+2 / m .
$$

Because this is true for every $m$ we have:

$$
\inf _{b \in B} d(b, y) \leq 2 L(X)
$$

(b) Follows from Corollary 3.7 and (a). I

Sometimes we can sharpen our inequalities, as the following theorem shows.

THEOREM 5.2 For a $p-M E T^{\infty}$ space $X$ with an ultrametric $d$ and $Y \subset X$ we have:

$$
\begin{aligned}
& \text { (a) } L(Y) \leq L(X) \\
& \text { (b) } S(Y) \leq S(X) .
\end{aligned}
$$

Proof. Simply note that Equation 10 in the proof of Theorem 5.1 now becomes

$$
d\left(y_{n, m}, y\right) \leq \max \left(d\left(y_{n, m}, c_{n}\right), d\left(c_{n}, y\right)\right) \leq L(X)+1 / m .
$$

For metrics which are not ultrametrics Theorem 5.1 cannot be improved, as the following counterexamples show.

COUNTEREXAMPLE 5.3 Consider $\mathbf{R}$ with the following metric:

$$
d(0, x)=d(x, 0)=1 / 2 \quad \forall x \in \mathbf{R} \text { and } d(x, y)=1_{x}(y) \text { where } x, y \in \mathbf{R}_{0} .
$$

Then $L\left(\mathbf{R}_{0}\right)=1$ but $L(\mathbf{R})=1 / 2$.

COUNTEREXAMPLE 5.4 Consider $\mathbf{R}$ with the following pseudo-quasi-metric:

$$
d_{n}(0, x)=1 / n \text { and } d_{n}(x, 0)=1-1 / n \quad \forall x \in \mathbf{R} \text { and } d(x, y)=1_{x}(y) \text { where } x, y \in \mathbf{R}_{0} .
$$

Then $L\left(\mathbf{R}_{0}\right)=1$ but $L(\mathrm{R})=1 / n$ and $S(\mathrm{R})=1-1 / n$.

So the foregoing results are not true for general $\mathrm{p}-(\mathrm{q})-M E T^{\infty}$ spaces.

6 PRODUCTS OF P-Q-MET ${ }^{\infty}$ SPACES.

For $p-q-M E T^{\infty}$ spaces we have:

PROPOSITION 6.1 Let $\left(X_{j}\right)_{j \in J}$ be an at most countable family of $p-q-M E T^{\infty}$ spaces. Then:

$$
L\left(\prod_{j \in J} X_{j}\right)=\sup _{j \in J} L\left(X_{j}\right)
$$

Proof. The first inequality follows from Remark 4.1. We now prove the second inequality. Put $X:=\prod_{, \in J} X$, and for each $X$, we consider $A_{j}$ countable such that:

$$
L(X,)=\sup _{x \in X_{j}} \inf _{a \in \Lambda_{j}} d_{j}(a, x) .
$$


Now put $A:=\prod_{, \in J} A$,

If $\mathrm{J}$ is finite then $A$ is countable and it is clear from equation 12 that:

$$
\begin{aligned}
L\left(\prod_{\jmath \in J} X,\right) & \leq \sup _{x \in X} \inf \max _{a \in A} d,\left(a_{\jmath}, x_{\jmath}\right) \\
& \leq \sup _{\jmath \in J} L\left(X_{j}\right) .
\end{aligned}
$$

If $\mathrm{J}$ is countable we can always take $\mathrm{J}=\mathrm{N}$. Let us remark first of all that $\mathrm{L}(\mathrm{A})=0$. Indeed, each set $A$, has a topological coreflection coarser than the discrete topology, hence the product topology is coarser than the product of the discrete topologies. But this last product is homeomorfic with $\mathrm{N}^{\mathrm{N}}$, which is Lindelof [9]. Hence the product-topology is also Lindelö which proves $L(A)=0$.

From equation 12 we know that for every $\varepsilon>0, i \in N$ and $z_{\mathbf{i}} \in X_{\mathbf{i}}$ there exists $a_{\mathbf{i}} \in A_{\mathbf{i}}$ such that $d_{\mathfrak{i}}\left(a_{\mathfrak{\imath}}, z_{\mathrm{i}}\right) \leq L\left(X_{\mathfrak{\imath}}\right)+\varepsilon$. Hence:

$$
\sup _{i \in \mathbb{N}} d_{i}\left(a_{i}, z_{i}\right) \leq \sup _{i \in \mathbf{N}} L\left(X_{i}\right)+\varepsilon
$$

In the following we shall consider with each $\left(z_{\imath}\right)_{1 \in J} \in X$ this $\left(a_{\jmath}\right)_{\jmath \in J} \in A$. We now have:

$$
\begin{aligned}
& L\left(\prod_{j \in N} X_{\jmath}\right) \leq \sup _{K: X \rightarrow 2^{(N)}} \inf _{Y \in 2^{2(X))}} \sup _{z \in X} \inf _{y \in Y} \sup _{\mathbf{i} \in K(\boldsymbol{y})} d_{i}\left(y_{i}, z_{i}\right) \\
& \leq \sup _{K: X \rightarrow 2^{2}(N)} \inf _{Y \in 2^{2(A))}} \sup _{z \in X} \inf _{y \in Y} \sup _{z \in K(y)} d_{i}\left(y_{1}, z_{z}\right) \\
& \leq \sup _{K: X \rightarrow 2^{(N)}} \inf _{Y \in 2^{((\Lambda))}} \sup _{z \in X} \inf _{y \in Y} \sup _{i \in K(y)}\left[d_{i}\left(y_{2}, a_{i}\right)+d_{i}\left(a_{i}, z_{i}\right)\right] \\
& \leq \sup _{K: X \rightarrow 2^{(N)}} \inf _{Y \in 2^{((A)))}} \sup _{a \in A} \inf _{y \in Y} \sup _{i \in K(y)} d_{i}\left(y_{i}, a_{i}\right)+\sup _{i \in N} L\left(X_{i}\right)+\varepsilon \\
& \leq L(A)+\sup _{i \in \mathbf{N}} L\left(X_{i}\right)+\varepsilon .
\end{aligned}
$$

Since the measures of separability and Lindelof coincide for metric spaces, and the measure of separability is stable for even some uncountable products. This could also be true for the measure of Lindelö. Unfortunately this is not the case as the following example shows:

COUNTEREXAMPLE 6.2 We know from [9] that $\mathrm{Z}^{\mathbf{R}}$ is a completely regular non-normal topological space. From [3] p.154 it then follows that the topological bicoreflection of $\mathbf{Z}^{\mathbf{R}}$ is not Lindelö. On the other hand we can consider $\mathbf{Z}$ with the Euclidean metric and $\mathbf{Z}^{\mathbf{R}}$ as the product. It is clear that $L(\mathbf{Z})=0$, but we shall show that $L\left(\mathbf{Z}^{\mathbf{R}}\right) \geq 1$. Indeed suppose that $L\left(\mathbf{Z}^{\mathbf{R}}\right)<1$ then consider in each $x \in X$ a neighborhood $V_{x}$. It is clear that for every $x$ we have a finite $K_{V(x)}$ such that $\cap_{\jmath \in K_{V(\varepsilon)}} \pi_{j}^{-1}\left(x_{\jmath}\right) \subset V_{x}$. Then we have:

$$
\sup _{K: X \rightarrow 2(\mathbf{R})} \inf _{\left.Y \in \mathbf{2}^{(}\left(\mathbf{Z}^{\mathbf{R}}\right)\right)} \sup _{\boldsymbol{z} \in \mathbf{Z}^{\mathbf{R}}} \inf _{\boldsymbol{x} \in Y} \sup _{j \in K(\boldsymbol{x})} d,(x, z)<1 .
$$

In particular for $K_{V}: X \rightarrow 2^{(\mathbf{R})}$ we can find $Y \in 2^{\left(\left(\mathbf{Z}^{\mathbf{R}}\right)\right)}$ such that for every $z \in \mathbf{Z}^{\mathbf{R}}$ there exists $x \in Y$ such that:

$$
\sup _{, \in K_{V(\bullet)}} d_{j}(x, z)<1
$$

Because the only distance in $\mathbf{Z}$ smaller than 1 is 0 , we deduce that: $\cup_{x \in Y} \cap_{j \in K_{v(=)}} \pi_{j}^{-1}(x)=,Z^{\mathbf{R}}$. But this means that $\mathbf{Z}^{\mathbf{R}}$ (as a topological space) is Lindelof which is not the case. Hence $L\left(\mathbf{Z}^{\mathbf{R}}\right) \geq 1$. 
REMARK 6.3 If we choose the distance function in $\mathrm{Z}$ such that the distance between two different points is always $N$, then with the same reasoning as above we can prove that $L\left(\mathbf{Z}^{\mathbf{R}}\right)>N$ and if we choose $a \infty-p$ metric such that the distance is $\infty$ for two different points then $L\left(\mathbf{Z}^{\mathbf{R}}\right)=\infty$.

We know that for general approach spaces the measure of separability for an uncountable (larger than the continium) product is not necessarily equal to the supremum of the measures of separability of its components. For products of p- $M E T^{\infty}$ spaces we have the following counterexample.

COUNTEREXAMPLE 6.4 Consider a set $U$ with cardinality larger than the continuum. Then as a product of discrete metric spaces $S\left(2^{U}\right)=0$ iff its topological coreflection is separable. Since this is not the case [2], and $\delta\left(X \times 2^{X}\right) \subset\{0,1\}$ we have $S\left(2^{U}\right)=1$.

\section{REFERENCES.}

[1] R. Baekeland, R. Lowen, 'Prohorov's theorem revisited', to appear.

[2] J. Dugundji, Topology, Allan and Bacon, Boston, 1966.

[3] Gaal, Point set topology, Academic Press, 1964.

[4] E. Lowen, R. Lowen, 'A quasi-topos containing CONV and MET as full subcategories', Internat. J. Math. \& Math. Sci., Vol 11 no 3 (1988) 417-438.

[5] R. Lowen, 'Kuratowski's measure of non-compactness revisited', Quart. J. Math. Oxford (2), 39 (1988), 235-254.

[6] R. Lowen, 'Approach spaces: a common supercategory of TOP and MET', Math. Nachr., 141 (1989),183-226.

[7] R. Lowen, 'Approximations of weak convergence', Math. Nachr. 160 (1993), 299-312.

[8] C. Kuratowski, 'Sur les espaces complets', Fund. Math. 15 (1930), 301-39.

[9] L.A. Steen and J.A. Seebach Jr, Counterexamples in Topology, Holt, Rinehart and Winston, Inc, 1970. 


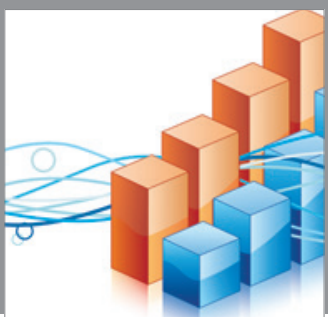

Advances in

Operations Research

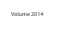

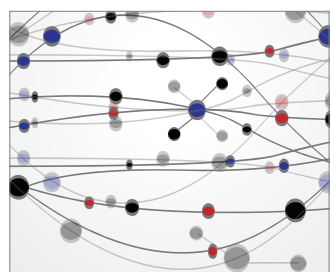

\section{The Scientific} World Journal
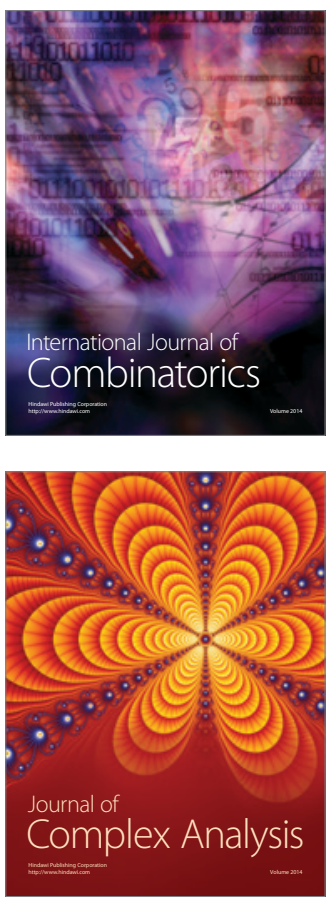

International Journal of

Mathematics and

Mathematical

Sciences
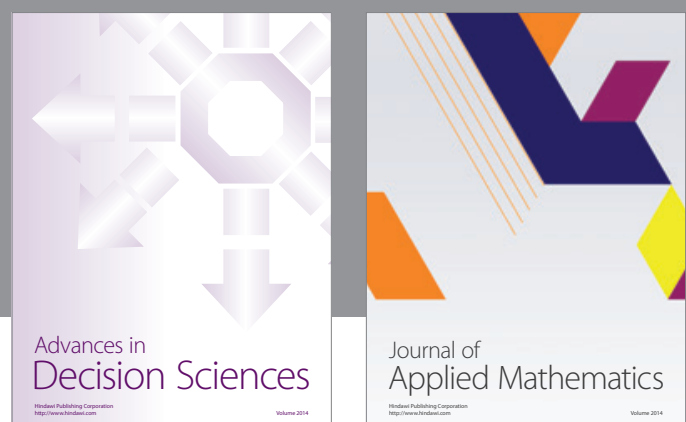

Journal of

Applied Mathematics
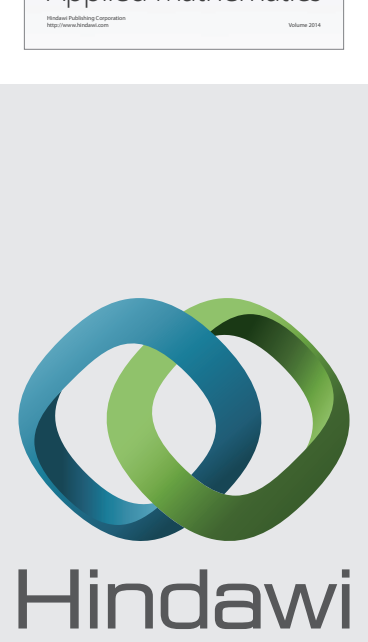

Submit your manuscripts at http://www.hindawi.com
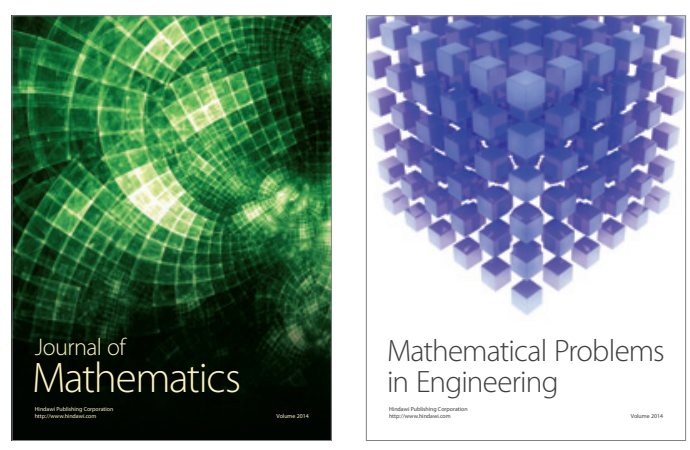

Mathematical Problems in Engineering
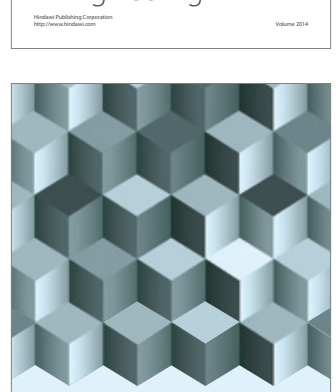

Journal of

Function Spaces
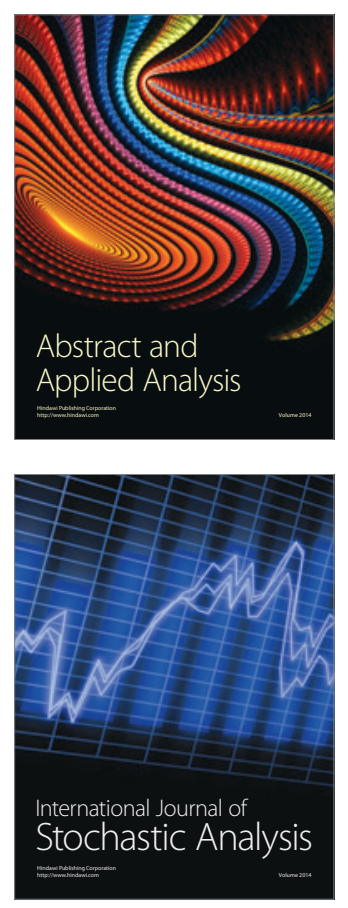

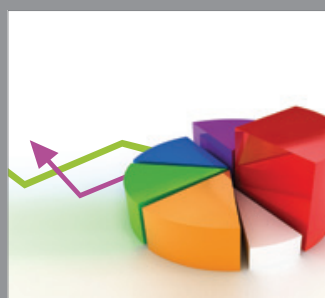

ournal of

Probability and Statistics

Promensencen
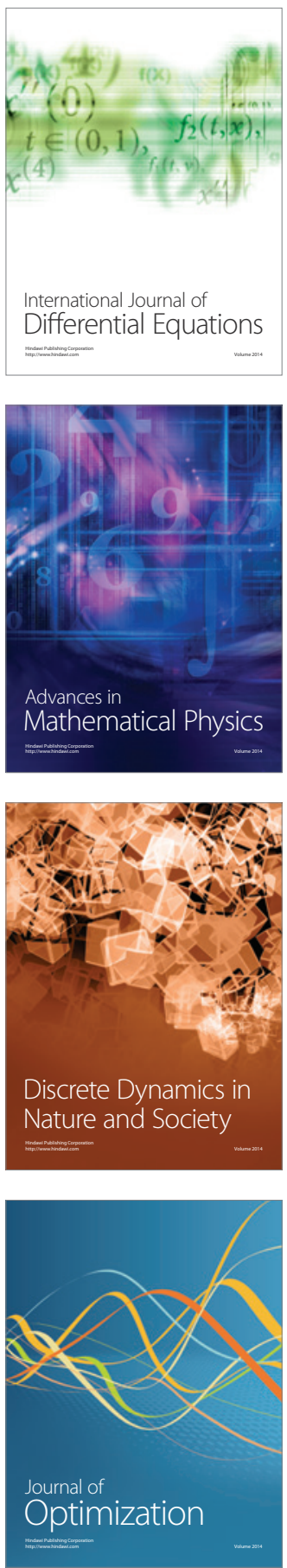